\title{
Review Article \\ Exercise-Induced Muscle Damage and Running Economy in Humans
}

\section{Cláudio de Oliveira Assumpção, Leonardo Coelho Rabello Lima, Felipe Bruno Dias Oliveira, Camila Coelho Greco, and Benedito Sérgio Denadai}

Human Performance Laboratory, UNESP, Avenue 24 A, Bela Vista-Rio, 13506-900 Rio Claro, SP, Brazil

Correspondence should be addressed to Benedito Sérgio Denadai; bdenadai@rc.unesp.br

Received 18 December 2012; Accepted 18 January 2013

Academic Editors: L. Guimarães-Ferreira, H. Nicastro, J. Wilson, and N. E. Zanchi

Copyright (c) 2013 Cláudio de Oliveira Assumpção et al. This is an open access article distributed under the Creative Commons Attribution License, which permits unrestricted use, distribution, and reproduction in any medium, provided the original work is properly cited.

\begin{abstract}
Running economy (RE), defined as the energy demand for a given velocity of submaximal running, has been identified as a critical factor of overall distance running performance. Plyometric and resistance trainings, performed during a relatively short period of time ( 15-30 days), have been successfully used to improve RE in trained athletes. However, these exercise types, particularly when they are unaccustomed activities for the individuals, may cause delayed onset muscle soreness, swelling, and reduced muscle strength. Some studies have demonstrated that exercise-induced muscle damage has a negative impact on endurance running performance. Specifically, the muscular damage induced by an acute bout of downhill running has been shown to reduce RE during subsequent moderate and high-intensity exercise $\left(>65 \% \mathrm{VO}_{2} \max \right)$. However, strength exercise (i.e., jumps, isoinertial and isokinetic eccentric exercises) seems to impair RE only for subsequent high-intensity exercise $\left(\sim 90 \% \mathrm{VO}_{2} \mathrm{max}\right)$. Finally, a single session of resistance exercise or downhill running (i.e., repeated bout effect) attenuates changes in indirect markers of muscle damage and blunts changes in RE.
\end{abstract}

\section{Introduction}

Running economy (RE), defined as the energy demand for a given velocity of submaximal running, is an important predictor of aerobic running performance, particularly in elite runners who have a similar aerobic power (i.e., maximal oxygen uptake, $\mathrm{VO}_{2} \max$ ) [1]. Runners with high $\mathrm{RE}$ demonstrate lower energetic cost at submaximal velocity and consequently tend to run faster at given distance or longer at a constant velocity.

A number of biomechanical (e.g., gait patterns, kinematics, and the kinetics of running) and physiological factors (e.g., oxidative muscle capacity) seem to influence RE in trained athletes $[2,3]$. Moreover, some interventions (plyometric, resistance and altitude training) performed during relatively short periods of time ( 15-30 days) have been successfully used to improve RE [4-6]. Plyometric and resistance trainings lead to neuromuscular adaptations such as increased neural drive to the muscles and changes in muscle stiffness and muscle fiber composition, which might reduce the energetic cost during submaximal exercise. However, plyometric and resistance trainings, especially when they are unaccustomed activities, may cause delayed onset muscle soreness (DOMS), swelling, and reduced muscle strength. The negative effect of muscle-damaging exercises on endurance running performance has been experimentally demonstrated in both animal $[7,8]$ and human experiments [9]. However, studies that have investigated the effect of exercise-induced muscle damage (EIMD) on RE have produced equivocal results [9-12]. This review discusses the effects of EIMD induced by different exercise types (strength, long-distance running, and downhill running) on RE. Different recovery strategies aiming to enhance the RE after EIMD are also addressed.

\section{Running Economy}

Aerobic fitness, as well as running performance, can be measured by different variables, for example, maximal oxygen uptake $\left(\mathrm{VO}_{2} \mathrm{max}\right)$, lactate threshold (LT), onset of blood 
lactate accumulation (OBLA), movement economy (ME), or running economy (RE). The $\mathrm{VO}_{2}$ max, which reflects an individual's maximal rate of aerobic energy expenditure, has been considered the gold standard for measuring aerobic power [13]. Indeed, the $\mathrm{VO}_{2} \max$ has a positive association with aerobic running performance obtained during middle- and long-distance events $(1,500 \mathrm{~m}-42,195 \mathrm{~m})$ [14-16]. However, some studies [17-20] have shown that subjects with similar $\mathrm{VO}_{2}$ max values may attain different aerobic running performance or $\mathrm{VO}_{2}$ values during exercise of similar duration and intensity. These differences are most likely due to variations in $\mathrm{ME}$ among subjects.

The ME is defined as the amount of energy necessary $\left(\mathrm{Kcal} \cdot \mathrm{min}^{-1}\right)$ to perform a given task [21]. However, due to the difficulty to determine the external work performed during running, RE, expressed as the volume of oxygen uptake $\left(\mathrm{mL} \cdot \mathrm{Kg}^{-1} \cdot \mathrm{min}^{-1}\right)$ during a specific submaximal running intensity $\left(\mathrm{Km} \cdot \mathrm{h}^{-1}\right)$, has been adopted. There is a strong association between $\mathrm{RE}$ and aerobic running performance, with $\mathrm{RE}$ being a better predictor of performance than $\mathrm{VO}_{2} \mathrm{max}$, particularly in athletes who have similar $\mathrm{VO}_{2} \max [19,22]$.

Several factors have been proposed to influence RE in trained subjects. These include oxidative muscle capacity and muscle stiffness. Muscle stiffness corresponds to the ability of the muscles to store and release elastic energy. Moreover, some interventions such as training, environment, and muscle damage can modify the oxygen cost over a range of running speeds $[6,11,23-25]$.

Improved oxidative muscle capacity can be associated with reduced oxygen consumption per mitochondrial respiratory chain during submaximal exercise. Trained subjects are known to have better RE than untrained individuals, and long-distance runners are more economical than middledistance runners [26]. Additionally, a high weekly volume of training has also been associated with better RE. However, a short period (4-6 weeks) of high-intensity aerobic training (near or above $\mathrm{VO}_{2} \max$ ) can also lead to improvement in the $\mathrm{RE}$ of trained runners [27].

In addition to aerobic characteristics and adaptations, neuromuscular profile factors have also been considered important aspects of RE. Type II muscle fibres seem to be positively correlated with submaximal energy consumption, especially at lower speeds [28]. Furthermore, both muscular stiffness and the ability to rapidly develop muscular force (i.e., rate of force development (RFD)) have demonstrated significant correlations with RE $[29,30]$. Stiffer muscletendon complexes may increase elastic energy storage by reducing the ground contact time, thus decreasing the running oxygen cost. Similar to stiffness, a higher RFD is associated with a shorter time to generate a contraction. This effect could also diminish the ground contact time and running oxygen cost. Heavy weight and plyometric training associated with endurance training have improved $\mathrm{RE}$ in well-trained runners [5,31]. Basically, these types of strength training induce neuromuscular adaptations such as increased neural drive to the muscles, altered muscle-tendon complex stiffness, and changed muscle fibre composition (i.e., I $\rightarrow$ IIA $\leftarrow$ IIX).
Environmental variables can also be used to reduce the energetic cost of running. RE can be improved (2-3\%) after relative short periods ( $15-20$ days) of altitude exposure $(\sim 2.000-4.500 \mathrm{~m})$. Altitude exposure during daily activities, sleeping, or training can enhance RE at sea level altitude through haematological and muscle changes in favour of oxygen transport [32-34]. Moreover, heat exposure during training sessions can also improve RE by enhancing the thermoregulatory process, thus reducing the cardiovascular and muscle work for a given exercise intensity $[23,35,36]$.

More recently, EIMD has also proposed to generate important modifications in RE. Muscular damage induced by an acute bout of downhill running has shown to reduce RE in the days following the intervention (24-120 hours) $[9,11]$. Specific aspects of this intervention are addressed hereafter.

\section{Muscle Damage and the Repeated Bout Effect}

Skeletal muscle damage has been considered an important factor contributing to DOMS and strength loss after eccentric exercise [37]. Basically, the exercise conditions at which muscle damage can be induced are unaccustomed exercises and exercises with higher intensity or longer duration than those to which the subject is adapted $[37,38]$. The resulting metabolic overload and mechanical strain have been suggested the main factors generating muscle damage [38]. Warren et al. [39] have suggested that measures of muscle function such as strength and power are effective indicators of both the magnitude and time course of muscle damage. Depending on the magnitude of muscle damage, muscle force at isometric, and dynamic testing conditions may be impaired for 1-7 days after the exercise [40-43]. Other important symptoms of muscle damage are disruption of the sarcolemma and extracellular matrix [44, 45], increased blood levels of creatine kinase (CK) and myoglobin (MB), stiffness, and swelling [46-48].

In general, muscle damage can be induced by both static (isometric) and dynamic (concentric and eccentric) muscle contractions. However, there is substantial evidence that eccentric muscle actions result in greater muscle damage than isometric or concentric actions [49-52]. The magnitude of strength loss after EIMD may vary between $5-10 \%$ and $~ 60 \%$ $[43,52]$, depending on the characteristics of the protocol and the type of muscle actions (i.e., isometric, concentric or eccentric) used during the posttest. The different effects of eccentric versus isometric or concentric actions have also been verified in the context of whole body exercises (i.e., running, cycling, and cross-country skiing) [43, 53]. For example, muscle damage and strength loss are higher during running ( 20-30\%), which involves concentric and eccentric actions, when compared with cycling $(\sim 10-15 \%)$, which involves mainly concentric actions [53]. In accordance with Millet and Lepers [53], although concentric and eccentric actions are present during cross-country skiing, muscular damage is considerably lesser during this exercise than in running because shock waves are present only during running. The main factors attributed to the greater effect of eccentric contractions on muscle damage are the higher peak torque 
values [54] and reduced motor unit activation for a given force [54-56], both of which induce a higher mechanical stress on the muscles [54]. Other important aspects of the greater muscle damage induced by eccentric muscle actions are that no energy (ATP) is necessary to detach the crossbridges formed during muscle contraction [57] and that the longer length of the muscles during the contraction generates greater muscle damage.

In addition to the main mechanical factors (i.e., the force level produced and the change in muscle length) [37, $58,59]$, some metabolic factors such as substrate depletion, calcium influx, and reactive oxygen species have also been proposed to influence muscle damage $[38,60]$. The effects of the different mechanical and metabolic factors that would contribute to muscle damage do not occur at the same time. The time course of the events involves damage in components of excitation-contraction system and sarcomeres [59] and degeneration and regeneration of muscle fibres, during which DOMS, stiffness, and swelling occur [37]. Additionally, there is an inflammatory response generating a transfer of fluid and cells to remove damaged contractile proteins and cellular debris from the damaged muscles [61]. Thereafter, the muscle regeneration process is initiated [61]. Although some of these effects may appear only some hours after the exercise, muscle strength may be impaired during and immediately after the exercise. Thus, mechanisms other than muscle damage can also explain the muscle fatigue (i.e., strength loss).

It has been suggested that the magnitude of muscle damage and the loss of muscle function might be attenuated after one bout of eccentric exercise [62-64]. This concept is known as the repeated bout effect (RBE). The RBE has been demonstrated after both eccentric muscle actions [65] and downhill running [66]. In general, this protective effect is confirmed by the reduced decrements and faster recovery of muscle strength, less swelling and DOMS, and attenuated changes in $\mathrm{CK}$ and $\mathrm{MB}$ in the blood $[62,67,68]$. In addition, alterations in muscle circumference or echo intensity (inflammation) are also smaller after the first eccentric exercise bout [68]. This protective effect has been demonstrated after a few days of the eccentric exercise [66] and may last up to 6 months (circumference, DOMS, and inflammation) or 9 months (maximal isometric force, CK), depending on the marker of muscle damage [65].

It has been hypothesized that the RBE is mediated by neural, cellular, and mechanical mechanisms $[63,64]$. The neural changes proposed to contribute to the RBE are increased slow-twitch fibre recruitment and synchronisation of motor unit firing, better distribution of the workload among muscle fibres, higher participation of synergist muscles to torque production, and increased motor unit activity relative to torque produced [69-71]. Neural mechanisms have been suggested based on the reduced median frequency [69], which reflects some central aspects related to motor unit recruitment. Howatson et al. [69] have demonstrated a $10 \%$ decrease in median frequency 14 days after a bout consisting of either 10 or 45 maximal eccentric actions. RBE has also been observed in the untrained contralateral limb, referred to as the contralateral RBE [72]. These studies $[69,72]$ confirm that, in addition to intramuscular adaptations, central aspects regarding motor unit recruitment are also involved in RBE.

The main mechanical adaptations associated with RBE are increased muscle stiffness and intramuscular connective tissue and changes in the intermediate filament system (maintenance of structural integrity of sarcomeres) [63]. Cellular adaptations are associated with higher number of sarcomeres in myofibrils $[59,73]$, which might decrease myofibrillar disruption in the next exercise bout, strengthened plasma membranes, increased protein synthesis, removal of stress-susceptible fibres $[59,74,75]$, and remodelling of the cytoskeleton, including effects on proteins such as titin and desmin, talin and vinculin [76], which might improve the strength and the stability of sarcomeres and protect muscle fibers against injuries. Other adaptation that has been hypothesized to explain the RBE is the lesser inflammatory response. Since the mechanical disruption is decreased after the first eccentric exercise bout, the stimulus for the inflammatory response is also reduced after the exercise [73, 74]. Some of these alterations have been associated with reduced muscle damage (strengthened extracellular matrix) and a change in the optimal angle for torque production toward a longer muscle length (increases in number of sarcomeres).

The magnitude of muscle damage induced by eccentric exercise is greater at longer muscle lengths $[65,77]$. When the muscles are elongated, the sarcomere length is also greater. Because the severity of muscle damage is influenced by the muscle strain generated $[73,78]$, it has been suggested that the RBE would be greater under conditions of longer muscle lengths. Nosaka et al. [73] have investigated the effect of the range of motion of the exercise used to induce muscle damage on the $\mathrm{RBE}$. The protocol used to induce muscle damage involved 24 maximal eccentric contractions of the elbow joint, using amplitudes of $50-100^{\circ}$ or $130-180^{\circ}$. Although the changes (maximal isometric strength, range of motion, upper arm circumference, muscle soreness, and CK) induced by the first bout were significantly greater using the higher amplitude, both exercise conditions induced RBE. However, the effect generated by the short range of motion was lesser than that promoted by the higher amplitude.

Other factor that can modify this protective effect (i.e., $\mathrm{RBE}$ ) of eccentric exercise is the magnitude of muscle damage $[65,66]$, which is influenced by the exercise intensity of the first bout. Chen et al. [66] showed that 30 eccentric contractions performed at $40 \%$ of maximal isometric strength generated a smaller attenuation of the changes in indirect markers of muscle damage (20-60\%) than maximal eccentric exercise (65-100\%).

It has been also demonstrated that both the muscle damage level (i.e., CK) and strength impairment and recovery (i.e., isometric torque) are progressively greater with increases in the number of bouts (1-4). However, Chen et al. [68] have demonstrated that repetitive submaximal eccentric exercise bouts (40\% MVC) performed every two weeks promote a protective effect similar to that induced by one maximal eccentric exercise bout. In this study, the main indirect markers of muscle damage were less affected by the second to the fourth bouts of submaximal eccentric exercise than the first; that is, the protective effect is promoted under 
TABLE 1: Comparison of the effects of the resistance exercise on running economy.

\begin{tabular}{|c|c|c|c|c|c|}
\hline Study & Subjects & EIMD & Muscle damage & $\mathrm{VO}_{2} \max (\%)$ & RE (\%) \\
\hline $\begin{array}{l}\text { Paschalis et al. } \\
{[10]}\end{array}$ & 10 healthy males & 120 eccentric actions & $\begin{array}{c}\uparrow \mathrm{CK}, \\
\uparrow \mathrm{DOMS} \text {, and } \\
\downarrow \text { ROM, and } \\
\downarrow \text { strength }\end{array}$ & 55 and 75 & $\sqrt{ }$ \\
\hline Burt et al. [12] & 9 healthy men & $\begin{array}{c}100 \text { squats at } 80 \% \\
\text { body mass }\end{array}$ & $\begin{array}{l}\sqrt{ } \mathrm{CK}, \\
\uparrow \mathrm{DOMS}, \text { and } \\
\downarrow \text { strength }\end{array}$ & 90 & $\downarrow 4-5$ \\
\hline Vassilis et al. [87] & $\begin{array}{l}24 \text { young healthy } \\
\text { men }\end{array}$ & 120 eccentric actions & $\begin{array}{c}\uparrow \mathrm{CK}, \\
\uparrow \mathrm{DOMS}, \\
\downarrow \text { strength }\end{array}$ & 70 & $\sqrt{ }$ \\
\hline Scott et al. [88] & $\begin{array}{l}8 \text { active men and } \\
8 \text { active women }\end{array}$ & $\begin{array}{c}3-4 \times 10 \text { repetitions } \\
\text { of squat, lunges, step } \\
\text { up and step down, } \\
\text { and stiff-legged } \\
\text { deadlift }\end{array}$ & $\uparrow \mathrm{DOMS}$ & 70 & $\sqrt{ }$ \\
\hline
\end{tabular}

EIMD: exercise-induced muscle damage; $\% \mathrm{VO}_{2}$ max: exercise intensity at which running economy was measured; RE: running economy; CK: creatine kinase; DOMS: delayed onset muscle soreness; ROM: range of motion; $\downarrow$ indicates decrease; $\sqrt{ }$ indicates no change; $\uparrow$ indicates increase.

conditions of reduced levels of induced muscle damage. Even after repeated submaximal bouts the magnitude of muscle damage was still smaller than that induced by one maximal bout. The authors suggested that the effect of exercise intensity on the protective effect of the first bout does not apply when some bouts of low-intensity exercise are performed. Therefore, the magnitude of muscle damage does not necessarily affect the protective effect of eccentric exercise. Moreover, Howatson et al. [69] have compared two protocols of maximal eccentric contractions to induce muscle damage with 45 or 10 contractions. After 14 days, subjects performed the same protocol with 45 contractions. Although the effect of the higher volume of the first bout on damage markers (CK, DOMS, and isometric torque) was greater, the protective effects of both protocols were similar. Therefore, the intensity of the first bout seems to be the main aspect of the magnitude of muscle damage and RBE.

Because one exercise bout is enough to generate the $\mathrm{RBE}$, some studies have also investigated whether resistance training could also reduce the effects of eccentric exercise on muscle damage markers $[67,79]$. Specifically, Newton et al. [67] found that resistance-trained subjects demonstrated smaller RBE when compared with untrained subjects. Moreover, Falvo et al. [79] did not find changes in indirect markers of muscle damage (maximal isometric torque and $\mathrm{CK}$ ) in resistance-trained men. The authors attributed the absence of RBE to a lack of neural adaptation. Thus, it is likely that strength training induces to adaptations that reduce the RBE.

The majority of studies that have analysed the RBE used relatively short time periods after the eccentric exercise (i.e., from approximately 7-14 days to 6-9 weeks). However, some studies [80, 81] have reported that the RBE induced by 24 maximal eccentric actions of the elbow flexors may last up to 6 months. Nosaka et al. [80] aimed to investigate the responses of the main indirect markers of muscle damage (CK, maximal isometric torque, DOMS, and swelling) five days after the eccentric exercise bout, with sessions six, nine, and twelve months apart. The main finding of this study was that the RBE for strength, swelling, DOMS, and CK lasted up to six months.

\section{Strength Exercise, Muscle Damage, and Running Economy}

A variety of studies have investigated the influence of EIMD and DOMS on neuromuscular performance indicators (i.e., strength and rate of force development) [82-84]. These studies verified that the isomeric peak torque is compromised immediately after the damaging exercise that causes DOMS, with a gradual recovery in subsequent days. The magnitude and the recovery rate from strength loss seem to be related to the training history of the muscle group. For instance, when performing maximal eccentric contractions, upper limb muscles (less active) demonstrate greater loss of strength (50-70\%) and slower recovery (60-90 days) when compared to lower limb muscles (locomotory muscles) $(20-30 \%$ and 10-30 days, resp.) $[37,85]$. However, only a few studies have investigated the effects of EIMD and DOMS on aerobic performance indexes (e.g., $\mathrm{VO}_{2} \mathrm{max}$, lactate response to exercise, $\mathrm{VO}_{2}$ kinetics, and movement economy) [86]. These studies analysed the effects of EIMD on RE $[10,12]$ and $\mathrm{VO}_{2}$ kinetics during submaximal cycling exercise [86]. In this context, studies that investigated the effects of strength exercises (i.e., jumps, isoinertial and isokinetic eccentric exercises) on $\mathrm{RE}$ will be addressed (Table 1).

Paschalis et al. [10] analysed the effects of eccentric exercises on indirect muscle damage markers (CK, DOMS, $\mathrm{ROM}$, and isometric force) and RE in active individuals who were not engaged in strength training programs. The eccentric exercise protocol consisted of $120(12 \times 10)$ maximal voluntary contractions (MVC) at an angular velocity of 1.05 $\mathrm{rad} \cdot \mathrm{s}^{-1}$. Although indirect muscle damage markers were significantly altered in the subsequent days $(24-72 \mathrm{~h})$, the $\mathrm{RE}$ (assessed at 55 and $75 \% \mathrm{VO}_{2} \max$ ) was not modified. Similar data were obtained by Vassilis et al. [87], who analysed 
TABLE 2: Comparison of the effects of the downhill running on running economy.

\begin{tabular}{|c|c|c|c|c|c|}
\hline Study & Subjects & EIMD & Muscle damage & $\mathrm{VO}_{2} \max (\%)$ & RE (\%) \\
\hline Chen et al. [11] & 50 male students & $\begin{array}{c}30 \mathrm{~min} \text { DHR at } \\
-15 \%\end{array}$ & $\begin{array}{c}\uparrow \mathrm{CK}, \\
\uparrow \mathrm{DOMS}, \\
\downarrow \text { strength, and } \\
\uparrow \mathrm{LDH}\end{array}$ & $\begin{array}{c}70,80 \text {, and } \\
90\end{array}$ & $\downarrow 5$ \\
\hline Hamill et al. [92] & $\begin{array}{l}10 \text { recreational } \\
\text { female runners }\end{array}$ & $\begin{array}{c}30 \min \text { DHR at } \\
-15 \%\end{array}$ & $\begin{array}{c}\uparrow \mathrm{CK}, \\
\uparrow \mathrm{DOMS}\end{array}$ & 80 & $\sqrt{ }$ \\
\hline $\begin{array}{l}\text { Braun and Dutto } \\
{[93]}\end{array}$ & $\begin{array}{l}9 \text { endurance trained } \\
\text { men }\end{array}$ & $\begin{array}{c}30 \min \mathrm{DHR} \text { at } \\
-10 \%\end{array}$ & $\uparrow \mathrm{DOMS}$ & 65,75 , and 85 & $\downarrow 3$ \\
\hline Chen et al. [94] & $\begin{array}{l}10 \text { soccer trained } \\
\text { men }\end{array}$ & $\begin{array}{c}30 \mathrm{~min} \text { DHR at } \\
-15 \%\end{array}$ & $\begin{array}{c}\uparrow \mathrm{CK}, \\
\uparrow \mathrm{DOMS}, \\
\downarrow \text { strength, and } \\
\uparrow \mathrm{MB}\end{array}$ & 65,75 , and 85 & $\downarrow 4-7$ \\
\hline
\end{tabular}

EIMD: exercise-induced muscle damage; DHR: downhill running; $\% \mathrm{VO}_{2}$ max: exercise intensity at which running economy was measured; RE: running economy; CK: creatine kinase; DOMS: delayed onset muscle soreness; MB: myoglobin; LDH: lactate dehydrogenase; $\downarrow$ indicates decrease; $\sqrt{ }$ indicates no change; $\uparrow$ indicates increase.

the effects of eccentric exercise $\left(120 \mathrm{MVC}\right.$ at a $\left.60^{\circ} \cdot \mathrm{s}^{-1}\right)$ on $\mathrm{RE}$ in recreational athletes with no previous experience in resistance training. The $\mathrm{RE}$ (assessed at $70 \% \mathrm{VO}_{2} \max$ ) was not changed 48 hours after the damaging bout. Therefore, EIMD induced by isokinetic eccentric contractions do not seem to interfere on RE measured at moderate intensities (55$\left.75 \% \mathrm{VO}_{2} \max \right)$.

Using closed kinetic-chain exercises, Scott et al. [88] have also analysed the effects of EIMD on RE. The volunteers performed a series of lower extremity resistance exercises designed to induce DOMS. RE was analysed at $70 \% \mathrm{VO}_{2} \max , 24-30$ hours after the EIMD. Although the subjects demonstrated a higher rate of perceived exertion values, RE was maintained unaltered throughout the days after EIMD. In another study, Marcora and Bosio [9] did not find any alteration in $\mathrm{RE}\left(70 \% \mathrm{VO}_{2} \max \right)$ after 100 drop jumps, although DOMS, CK, and knee extensors strength were significantly affected by EIMD. Therefore, the evidence suggests that muscle damage induced by both open and closed kinetic-chain exercises dose not alter $\mathrm{RE}$ at moderate intensities (55-75\% $\left.\mathrm{VO}_{2} \max \right)$.

However, in a recent study, Burt et al. [12] presented conflicting data regarding the effect of EIMD on RE. In this study, indirect markers of muscle damage and RE were measured, $24-48 \mathrm{~h}$ after EIMD (10 sets of 10 squats at $80 \%$ body mass). Significant increases in all indirect markers of muscle damage, kinematic parameters (stride length and stride frequency), and oxygen uptake during submaximal running $\left(\sim 90 \% \mathrm{VO}_{2} \max \right)$ were observed at $24-48 \mathrm{~h}$ following the initial bout of EIMD. Some authors [82, 89] have suggested that the changes in $\mathrm{RE}$ are associated with decrements in neuromuscular function (i.e., MVC) after EIMD. However, both the magnitude and the time course of the changes in muscular function (MVC) and RE can be different. Therefore, changes during submaximal exercise (i.e., RE) may not be strictly associated with neuromuscular function.

As a whole, these data suggest that the effects of muscle damage induced by strength exercise (i.e., jumps, isoinertial and isokinetic eccentric exercises) on RE are intensityddependent. During moderate exercises (55-75\% $\mathrm{VO}_{2} \max$ ), $\mathrm{RE}$ is not altered by EIMD. However, during high-intensity exercise ( $\left.90 \% \mathrm{VO}_{2} \max \right), \mathrm{RE}$ is impaired. During high intensity exercise, additional type II fibres, which are the most affected by EIMD, are recruited. Moreover, at these intensities, the $\mathrm{VO}_{2}$ either attains a delayed steady state (heavy domain) or continues to increase slowly (i.e., $\mathrm{VO}_{2}$ slow component $\left(\mathrm{VO}_{2} \mathrm{SC}\right)$ ) reaching its maximal values at the end of exercise (severe domain) [90]. Although the physiological determinants of $\mathrm{VO}_{2} \mathrm{SC}$ remain poorly understood, some authors have proposed that an increased ATP and/or $\mathrm{O}_{2}$ cost of power production in fatigued fibres, rather than the additional recruitment of poorly efficient muscle fibres, is responsible for the $\mathrm{VO}_{2} \mathrm{SC}$ [91]. Therefore, the effects of strength exercise-induced muscle damage on RE seem to depend on the fibre recruitment pattern and/or on the mechanisms determining the $\mathrm{VO}_{2} \mathrm{SC}$.

\section{Downhill and Long-Distance Running, Muscle Damage, and Running Economy}

Adopting a more specific approach, some studies have investigated the influence of muscle damage induced by strenuous exercise (e.g., long-distance running) or downhill running on neuromuscular parameters, and RE. This aspect and the effect of some interventions on RE during the recovery period after EIMD are also addressed in this topic (Table 2).

As mentioned previously, muscle damage is usually induced by maximal and submaximal eccentric contractions, but it can also be observed when a high volume of eccentric/concentric contractions are performed, due to the eccentric contractions per se [95] or because of metabolite accumulation that may lead to stress and impairment of the muscle fibres [96]. Because a high number of concentric and, particularly, eccentric contractions are performed during long-distance running, the symptoms of muscle damage 
are usually observed immediately and a few days after the running bout.

In a study conducted by Millet et al. [97], changes in muscle function and muscle damage markers from 22 experienced marathon runners were collected and analysed after they had run an international extreme mountain ultramarathon. The race consisted of a $166 \mathrm{~km}$ marathon through mountainous terrain with the final destination set at $9500 \mathrm{~m}$ below the starting point. This predominately downhill configuration required a high number of eccentric contractions particularly for the knee extensors. Indirect muscle damage markers (strength, $\mathrm{CK}, \mathrm{LDH}$, and $\mathrm{MB}$ ) were analysed before, immediately after, and 2, 5, 7, 9, and 16 days after the marathon. The authors found higher decreases in force production immediately after the ultramarathon, most likely because of the fatigue experienced during the race. However, some of the strength markers remained altered until 5 days after exercise, as usually occurs after muscle damaging activities. Blood markers also demonstrated the highest value immediately after the race, returning to baseline values 5 days later. The authors found that even though this type of activity can induce extreme muscle damage, after 16 days, all the alterations induced by muscle damage and/or fatigue had returned to normal. Considering that force production is intimately related to RE, these findings may indicate that an extremely damaging activity may induce high levels of force loss and decreases in RE. Force production is usually fully recovered 5 days after the damaging activity. However, RE may recover at a faster rate than force.

To investigate factors that could influence RE, Kyrolainen et al. [89] subjected 7 experienced runners to a protocol simulating a marathon. RE and kinematic variables (stride length and frequency, mean contact time, external mechanical work and power, and angular displacements and velocities of the hip, knee, and ankle joints) were collected before, during (at the 1st, 13th, 26th, and 42nd kilometres), two hours after, and in the days $(2,4$, and 6$)$ after the marathon. Muscle damage markers (CK and SOR) were also collected after the exercise. The impairment of RE (i.e., higher oxygen consumption) was observed only at the end of the marathon (42nd kilometre and two hours afterward). CK and SOR were significantly increased immediately after the marathon and returned to baseline values only at the 6-day postexercise time point. These data may indicate that alterations in RE after marathon running may not be exclusively in the result of muscle damage but may be affected by other factors such as thermal stress. To better understand the time course of recovery of the various parameters of muscle function following marathon running, it is important to investigate other indirect EIMD markers, such as force and inflammatory response.

Muscle damage induced by downhill running has also been widely studied in the last decades. This type of exercise has been proven to induce muscle damage even when performed for relatively short periods (e.g., 30 minutes) due to higher mechanical stress applied to the lower limb muscles during the contact with the ground phase [95]. Some studies have shown that downhill running can lead to muscle damage of the same magnitude as plyometric or maximal eccentric exercises [92, 98]. Considering that downhill running induces muscle damage, a series of studies have investigated its influence on neuromuscular and metabolic markers in animals [99] and humans [11, 93, 94, 98]. In animals, downhill running has been utilised to induce overtraining [100] as well as a training method to increase the number of sarcomeres [101]. In humans, this exercise model has been recently studied in attempt to understand its influence on specific running and aerobic parameters, such as $\mathrm{RE}[93,98]$ and running kinematics.

To the best of our knowledge, Hamill et al. [92] performed the first study investigating the influence of downhill running on RE. In this study, 10 recreational female runners underwent a $30 \mathrm{~min}$ downhill running bout (DRB) with $-15 \%$ slope at $73.5 \%$ of maximal heart rate. Indirect markers of muscle damage (SOR and CK), RE ( $80 \% \mathrm{VO}_{2}$ max), and kinematic parameters were measured before and 2 and 5 days after the DRB. SOR and CK levels increased 2 days after the $\mathrm{DRB}$, returning to baseline values 5 days after the exercise. Although kinematic parameters were modified, the DRB did not alter RE. The authors proposed that changes in kinematics might be due to increases in SOR, which compromises the range of motion, and thus alters the movement patterns.

In another model to investigate the influence of downhill running-induced muscle damage on RE, Braun and Dutto [93] conducted a study in which 9 endurance-trained subjects underwent a DRB (30 minutes at $70 \% \mathrm{VO}_{2 \text { peak }}$ with a $-10 \%$ slope). Assessments of SOR, RE (65\%, $75 \%$ and $85 \%$ $\left.\mathrm{VO}_{2} \max \right)$, and stride length were performed before and 48 hours after the DRB. SOR was increased and RE was impaired 48 hours after the DRB, suggesting that muscle damage might have increased the energy cost of running. The authors stated that the muscle damage decreased the range of motion and strength, thus compromising running kinematics, which is known to be related to RE.

To better describe the time course of changes in RE, Chen et al. [94] subjected 10 soccer-trained volunteers to a downhill running protocol similar to that proposed by Braun and Dutto [93]. Muscle damage (MVC, SOR, CK and MB) and $\mathrm{RE}\left(65 \%, 75 \%\right.$, d and $\left.85 \% \mathrm{VO}_{2} \max \right)$ were assessed before and 1 hour and 1-5 days after the DRB. Alterations in muscle damage markers were consistent with those found in the literature, including increases in $\mathrm{CK}, \mathrm{MB}$, and $\mathrm{SOR}$, with peak values attained 48 hours after the DRB. Strength loss was also maximal immediately after the DRB. All muscle damage markers returned to baseline values 5 days after the DRB. The magnitude of change was smaller, and the time course recovery was faster for RE (4-7\% and 4 days, resp.) than for the indirect markers (i.e., isometric peak torque (IPT)) of muscle damage (7-21\% and 4 days, resp.). The authors suggested that the alterations in running kinematics, the need to recruit more muscle fibres, the impairment in the stretchshortening cycle, and the reduced levels of muscle glycogen might impair RE following a DRB.

Because alterations in the muscular tissue due to EIMD have been shown to affect RE because of differences in muscle fibre recruitment and other neuromuscular properties, Chen et al. [11] assessed RE at 3 different intensities (70, 80, and $\left.90 \% \mathrm{VO}_{2 \text { peak }}\right)$ after a DRB. Muscle damage markers showed the expected alterations, peaking 2 days after DRB. The 
alteration in $\mathrm{RE}$ measured at $90 \% \mathrm{VO}_{2 \text { peak }}$ was significantly higher than at $80 \% \mathrm{VO}_{2 \text { peak }}$. No significant change in $\mathrm{RE}$ was found at $70 \% \mathrm{VO}_{2 \text { peak }}$. Previous studies have indicated that fast-twitch motor units are progressively recruited with increased levels of exercise intensity [102]. Because several investigations have reported selective damage to type II muscle fibres after eccentric muscle actions in humans [40, 103], there appears to be a relationship between the motor unit recruitment pattern and impairment in RE.

Therefore, the effects of strength exercises and downhill running on RE seem to be different. While strength exercises seem to affect RE only during high intensity submaximal exercises ( $\left.\sim 90 \% \mathrm{VO}_{2} \max \right)$, downhill running also increases the energetic cost during moderate exercises $(>65 \%$ $\left.\mathrm{VO}_{2} \max \right)$. It is important to note that during running exercise, the $\mathrm{VO}_{2} \mathrm{SC}$ is attenuated (heavy intensity) and/or nonexistent (moderate). Thus, the effect of strength exercises on RE seems to occur only at running intensities at which the $\mathrm{VO}_{2} \mathrm{SC}$ is present. Greater muscle mass and/or the magnitude or specificity of muscle damage induced by downhill running may partially explain these results.

A variety of interventions have been proposed to enhance recovery from EIMD, that is, to reduce the severity and duration of injury and SOR. It is a common belief that lowintensity training (i.e., active recovery) enhances the recovery process by accelerating the return to homeostasis after EIMD. To investigate whether submaximal running would influence the recovery from DRB, Chen et al. [98] analysed the effect of 30-minute daily running exercises performed at different intensities $\left(40 \%, 50 \%, 60 \%\right.$, and $\left.70 \% \mathrm{VO}_{2 \text { peak }}\right)$ by different groups on the recovery of muscle damage and RE. Muscle damage was induced by a $\mathrm{DRB}$ (30 minutes at $70 \% \mathrm{VO}_{2 \text { peak }}$ with $-10 \%$ slope). The authors found that the time-course recovery of muscle damage markers and RE was similar for all groups, regardless of whether submaximal running was performed. Thus, low-to-moderate-intensity running seems not to improve the recovery from muscle damage and/or RE impairment.

Performing a similar subsequent bout of eccentric exercise results in significantly less change in the markers of muscle damage. This phenomenon is known as the RBE [104]. In fact, Byrnes et al. [105] and Chen et al. [66] demonstrated that when a DRB was repeated 1-6 weeks after the first bout, the indirect markers of muscle damage (isometric peak torque, CK, SOR, and range of motion) were significantly reduced. Moreover, Chen et al. [106] verified that the RBE was also observed in RE and running kinematics parameters. In this study, 12 male subjects underwent the same downhill running protocol adopted by Chen et al. [11] except the interval allowed between bouts that was twice as long, to allow full recovery from the first bout. The authors found significant changes in all markers of muscle damage after both protocols. However, the RE, kinematics parameters, and SOR were less affected after the second DRB. Therefore, one bout of DRB might induce a protective effect, leading to reduced levels of SOR and blunted changes in RE and biomechanical parameters.

In another study, Burt et al. [12] subjected 9 subjects to repeated bouts of 100 squats and measured muscle damage markers (isometric peak torque, vertical jump height, CK, and SOR) and RE before, immediately after the bouts, and 1-2 days after the bouts. The bouts were separated by enough time to recover from the EIMD symptoms. All muscle damage and RE markers were significantly affected by the first bout. However, no alterations in some muscle damage markers and RE were observed after the second bout. Thus, a previous damaging activity leads to blunted or nonexistent alterations in muscle damage markers and RE.

\section{Supplementation and Muscle Damage Recovery}

A series of recent studies has investigated the influence of different types of supplementation on recovery from and prevention of muscle damage. The main supplements that seem to protect against muscle damage are the flavonoids, which are known for their efficient anti-inflammatory and antioxidant properties. Studies investigating supplementation with flavonoid rich substances and their influence on muscle damage will be discussed.

Howatson et al. [107] conducted a study in which muscle damage, inflammatory response, and oxidative stress were measured before, immediately after, and 24 and 48 hours after a marathon. The purpose of this study was to investigate whether a tart cherry juice supplement would affect recovery from muscle damage after marathon running in 20 recreational marathon runners, using a double-blind placebo intervention. Muscle damage markers determined in this study were CK, LDH, DOMS, and IPT. Other parameters (total antioxidant status, thiobarbituric acid reactive species, $\mathrm{d}$ and protein carbonyls) were measured to identify inflammatory response, and oxidative stress. Both groups (control versus supplemented) demonstrated similar decreases in IPT. However, IPT was higher for the supplementation group at all time points, showing faster recovery. Moreover, the authors found that the supplementation enhanced the antiinflammatory response as well as reduced the oxidative stress.

Kuehl et al. [108] investigated the effects of tart cherry juice supplementation on SOR immediately after a longdistance running $(\sim 26 \mathrm{~km})$ bout. The study was performed in a randomised, double-blind placebo fashion in 54 experienced runners. The subjects were separated in two groups: placebo and tart cherry supplement. Both groups started ingesting their supplements 7 days prior to the running bout. The increase in SOR was significantly greater for the placebo group when compared to the tart cherry group. These findings are similar to those of Howatson et al. [107] and indicate that the anti-inflammatory and antioxidant properties of the tart cherry supplement might reduce SOR after EIMD.

Supplements containing flavonoid compounds have been shown to confer a protective effect against muscle damage either by attenuating a vast number of markers or by accelerating their recovery after the EIMD. This type of protection has been hypothesised to be due to the anti-inflammatory and antioxidant properties present in these types of compounds [107]. A number of studies have shown a direct relationship between flavonoid supplementation and protection against 
muscle damage. Because muscle damage may affect RE, it would be interesting to analyse if this type of supplementation can protect against RE impairment after EIMD.

\section{Conclusion}

Despite the systematic implications of RE on aerobic running performance, only a few experiments have specifically studied the response of this index after EIMD. Recent studies have analysed RE after strength exercises (i.e., jumps, isoinertial and isokinetic eccentric exercises) and downhill running. These studies have found that the magnitude of reduction in muscle function (MVC) after EIMD is greater than the RE and kinematic parameters. Moreover, the time course for the changes in muscle function, RE, and kinematic parameters are not similar. As a whole, these data suggest that the putative mechanisms underlying muscle function and RE during the recovery from EIMD are not completely shared. The effects of muscle damage on RE seem to depend of the interaction between the type of eccentric exercise and the intensity at which the RE is measured. Strength exercises seem to modify RE preferentially during high-intensity exercise $(\sim$ $\left.90 \% \mathrm{VO}_{2} \max \right)$. However, the effects of downhill running can also be observed at moderate intensities ( $\left.>65 \% \mathrm{VO}_{2} \mathrm{max}\right)$. Finally, a single session of strength exercise or downhill running attenuates changes in indirect markers of muscle damage and blunts changes in RE.

\section{Acknowledgments}

This research was supported by grants from Conselho Nacional de Desenvolvimento Científico e Tecnológico and Fundação de Amparo a Pesquisa do Estado de São Paulo, and the authors declar that they have no conflict of interests.

\section{References}

[1] J. Daniels and N. Daniels, "Running economy of elite male and elite female runners," Medicine and Science in Sports and Exercise, vol. 24, no. 4, pp. 483-489, 1992.

[2] T. Anderson, "Biomechanics and running economy," Sports Medicine, vol. 22, no. 2, pp. 76-89, 1996.

[3] P. U. Saunders, D. B. Pyne, R. D. Telford, and J. A. Hawley, "Factors affecting running economy in trained distance runners," Sports Medicine, vol. 34, no. 7, pp. 465-485, 2004.

[4] A. M. Turner, M. Owings, and J. A. Schwane, "Improvement in running economy after 6 weeks of plyometric training," Journal of Strength and Conditioning Research, vol. 17, no. 1, pp. 60-67, 2003.

[5] L. G. A. Guglielmo, C. C. Greco, and B. S. Denadai, "Effects of strength training on running economy," International Journal of Sports Medicine, vol. 30, no. 1, pp. 27-32, 2009.

[6] P. U. Saunders, R. D. Telford, D. B. Pyne, A. G. Hahn, and C. J. Gore, "Improved running economy and increased hemoglobin mass in elite runners after extended moderate altitude exposure," Journal of Science and Medicine in Sport, vol. 12, no. 1, pp. 67-72, 2009.

[7] M. D. Carmichael, J. M. Davis, E. A. Murphy et al., "Recovery of running performance following muscle-damaging exercise: relationship to brain IL-1 $\beta$," Brain, Behavior, and Immunity, vol. 19, no. 5, pp. 445-452, 2005.

[8] M. D. Carmichael, J. M. Davis, E. A. Murphy et al., "Role of brain IL-1 $\beta$ on fatigue after exercise-induced muscle damage," American Journal of Physiology, vol. 291, no. 5, pp. R1344-R1348, 2006.

[9] S. M. Marcora and A. Bosio, "Effect of exercise-induced muscle damage on endurance running performance in humans," Scandinavian Journal of Medicine and Science in Sports, vol. 17, no. 6, pp. 662-671, 2007.

[10] V. Paschalis, Y. Koutedakis, V. Baltzopoulos, V. Mougios, A. Z. Jamurtas, and V. Theoharis, "The effects of muscle damage on running economy in healthy males," International Journal of Sports Medicine, vol. 26, no. 10, pp. 827-831, 2005.

[11] T. C. Chen, K. Nosaka, M. J. Lin, H. L. Chen, and C. J. Wu, "Changes in running economy at different intensities following downhill running," Journal of Sports Sciences, vol. 27, no. 11, pp. 1137-1144, 2009.

[12] D. Burt, K. Lamb, C. Nicholas, and C. Twist, "Effects of repeated bouts of squatting exercise on sub-maximal endurance running performance," European Journal of Applied Physiology, vol. 113, no. 2, pp. 285-293, 2013.

[13] A. M. Jones and H. Carter, "The effect of endurance training on parameters of aerobic fitness," Sports Medicine, vol. 29, no. 6, pp. 373-386, 2000.

[14] P. A. Farrell, J. H. Wilmore, and E. F. Coyle, "Plasma lactate accumulation and distance running performance," Medicine and Science in Sports and Exercise, vol. 11, no. 4, pp. 338-344, 1979.

[15] R. R. Pate, C. A. Macera, S. P. Bailey, W. P. Bartoli, and K. E. Powell, "Physiological, anthropometric, and training correlates of running economy," Medicine and Science in Sports and Exercise, vol. 24, no. 10, pp. 1128-1133, 1992.

[16] D. W. Morgan and J. T. Daniels, "Relationship between $\mathrm{VO}_{2}$ max and the aerobic demand of running in elite distance runners," International Journal of Sports Medicine, vol. 15, no. 7, pp. 426429, 1994.

[17] E. F. Coyle, A. R. Coggan, M. K. Hopper, and T. J. Walters, "Determinants of endurance in well-trained cyclists," Journal of Applied Physiology, vol. 64, no. 6, pp. 2622-2630, 1988.

[18] D. L. Costill, H. Thomason, and E. Roberts, "Fractional utilization of the aerobic capacity during distance running," Medicine and Science in Sports and Exercise, vol. 5, no. 4, pp. 248-252, 1973.

[19] D. L. Conley and G. S. Krahenbuhl, "Running economy and distance running performance of highly trained athletes," Medicine and Science in Sports and Exercise, vol. 12, no. 5, pp. 357-360, 1980.

[20] D. W. Morgan, P. E. Martin, G. S. Krahenbuhl, and F. D. Baldini, "Variability in running economy and mechanics among trained male runners," Medicine and Science in Sports and Exercise, vol. 23, no. 3, pp. 378-383, 1991.

[21] W. A. Sparrow and K. M. Newell, "Metabolic energy expenditure and the regulation of movement economy," Psychonomic Bulletin and Review, vol. 5, no. 2, pp. 173-196, 1998.

[22] D. W. Morgan, P. E. Martin, and G. S. Krahenbuhl, "Factors affecting running economy," Sports Medicine, vol. 7, no. 5, pp. 310-330, 1989.

[23] L. W. Armstrong and C. M. Maresh, "The induction and decay of heat acclimatisation in trained athletes," Sports Medicine, vol. 12 , no. 5 , pp. 302-312, 1991. 
[24] P. U. Saunders, R. D. Telford, D. B. Pyne et al., "Shortterm plyometric training improves running economy in highly trained middle and long distance runners," Journal of Strength and Conditioning Research, vol. 20, no. 4, pp. 947-954, 2006.

[25] Ø. Støren, J. Helgerud, E. M. Støa, and J. Hoff, "Maximal strength training improves running economy in distance runners," Medicine and Science in Sports and Exercise, vol. 40, no. 6, pp. 1087-1092, 2008.

[26] L. J. Brandon, "Physiological factors associated with middle distance running performance," Sports Medicine, vol. 19, no. 4, pp. 268-277, 1995.

[27] B. S. Denadai, M. J. Ortiz, C. C. Greco, and M. T. De Mello, "Interval training at $95 \%$ and $100 \%$ of the velocity at $\mathrm{VO}_{2}$ max: effects on aerobic physiological indexes and running performance," Applied Physiology, Nutrition and Metabolism, vol. 31, no. 6, pp. 737-743, 2006.

[28] C. Bosco, G. Montanari, R. Ribacchi et al., "Relationship between the efficiency of muscular work during jumping and the energetics of running," European Journal of Applied Physiology and Occupational Physiology, vol. 56, no. 2, pp. 138-143, 1987.

[29] J. R. Fletcher, S. P. Esau, and B. R. MacIntosh, "Changes in tendon stiffness and running economy in highly trained distance runners," European Journal of Applied Physiology, vol. 110, no. 5, pp. 1037-1046, 2010.

[30] A. Arampatzis, G. De Monte, K. Karamanidis, G. Morey-Klapsing, S. Stafilidis, and G. P. Brüggemann, "Influence of the muscle-tendon unit's mechanical and morphological properties on running economy," The Journal of Experimental Biology, vol. 209, no. 17, pp. 3345-3357, 2006.

[31] R. W. Spurrs, A. J. Murphy, and M. L. Watsford, "The effect of plyometric training on distance running performance," European Journal of Applied Physiology, vol. 89, no. 1, pp. 1-7, 2003.

[32] B. D. Levine and J. Stray-Gundersen, "'Living high-training low: effect of moderate-altitude acclimatization with low-altitude training on performance," Journal of Applied Physiology, vol. 83, no. 1, pp. 102-112, 1997.

[33] E. Y. Robertson, P. U. Saunders, D. B. Pyne, C. J. Gore, and J. M. Anson, "Effectiveness of intermittent training in hypoxia combined with live high/train low," European Journal of Applied Physiology, vol. 110, no. 2, pp. 379-387, 2010.

[34] J. P. Wehrlin, P. Zuest, J. Hallén, and B. Marti, "Live high-train low for 24 days increases hemoglobin mass and red cell volume in elite endurance athletes," Journal of Applied Physiology, vol. 100, no. 6, pp. 1938-1945, 2006.

[35] J. Svedenhag, "Running economy," in Running and Science, J. Bangsbo and H. Larsen, Eds., pp. 85-105, Munksgaard, Copenhagen, Denmark, 2000.

[36] J. A. Houmard, D. L. Costill, J. A. Davis, J. B. Mitchell, D. D. Pascoe, and R. A. Robergs, "The influence of exercise intensity on heat acclimation in trained subjects," Medicine and Science in Sports and Exercise, vol. 22, no. 5, pp. 615-620, 1990.

[37] C. Byrne, C. Twist, and R. Eston, "Neuromuscular function after exercise-induced muscle damage theoretical and applied implications," Sports Medicine, vol. 34, no. 1, pp. 49-69, 2004.

[38] J. C. Tee, A. N. Bosch, and M. I. Lambert, "Metabolic consequences of exercise-induced muscle damage," Sports Medicine, vol. 37, no. 10, pp. 827-836, 2007.

[39] G. L. Warren, D. A. Lowe, and R. B. Armstrong, "Measurement tools used in the study of eccentric contraction-induced injury," Sports Medicine, vol. 27, no. 1, pp. 43-59, 1999.
[40] J. Friden, M. Sjostrom, and B. Ekblom, "Myofibrillar damage following intense eccentric exercise in man," International Journal of Sports Medicine, vol. 4, no. 3, pp. 170-176, 1983.

[41] M. J. Gibala, J. D. MacDougall, M. A. Tarnopolsky, W. T. Stauber, and A. Elorriaga, "Changes in human skeletal muscle ultrastructure and force production after acute resistance exercise," Journal of Applied Physiology, vol. 78, no. 2, pp. 702-708, 1995.

[42] T. Hortobágyi, J. Houmard, D. Fraser, R. Dudek, J. Lambert, and J. Tracy, "Normal forces and myofibrillar disruption after repeated eccentric exercise," Journal of Applied Physiology, vol. 84, no. 2, pp. 492-498, 1998.

[43] C. Byrne, R. G. Eston, and R. H. T. Edwards, "Characteristics of isometric and dynamic strength loss following eccentric exercise-induced muscle damage," Scandinavian Journal of Medicine and Science in Sports, vol. 11, no. 3, pp. 134-140, 2001.

[44] W. T. Stauber, P. M. Clarkson, V. K. Fritz, and W. J. Evans, "Extracellular matrix disruption and pain after eccentric muscle action," Journal of Applied Physiology, vol. 69, no. 3, pp. 868-874, 1990.

[45] J. Fridén and R. L. Lieber, "Eccentric exercise-induced injuries to contractile and cytoskeletal muscle fibre components," Acta Physiologica Scandinavica, vol. 171, no. 3, pp. 321-326, 2001.

[46] J. B. Rodenburg, P. R. Bar, and R. W. De Boer, "Relations between muscle soreness and biochemical and functional outcomes of eccentric exercise," Journal of Applied Physiology, vol. 74, no. 6, pp. 2976-2983, 1993.

[47] D. B. Pyne, "Exercise-induced muscle damage and inflammation: a review," Australian Journal of Science and Medicine in Sport, vol. 26, no. 3-4, pp. 49-58, 1994.

[48] T. C. Chen, H. L. Chen, A. J. Pearce, and K. Nosaka, "Attenuation of eccentric exercise-induced muscle damage by preconditioning exercises," Medicine and Science in Sports and Exercise, vol. 44, no. 11, pp. 2090-2098, 2012.

[49] P. V. Komi and J. T. Viitasalo, "Changes in motor unit activity and metabolism in human skeletal muscle during and after repeated eccentric and concentric contractions," Acta Physiologica Scandinavica, vol. 100, no. 2, pp. 246-254, 1977.

[50] P. M. Clarkson, W. C. Byrnes, K. M. McCormick, L. P. Turcotte, and J. S. White, "Muscle soreness and serum creatine kinase activity following isometric, eccentric, and concentric exercise," International Journal of Sports Medicine, vol. 7, no. 3, pp. 152-155, 1986.

[51] K. Vissing, K. Overgaard, A. Nedergaard, A. Fredsted, and P. Schjerling, "Effects of concentric and repeated eccentric exercise on muscle damage and calpain-calpastatin gene expression in human skeletal muscle," European Journal of Applied Physiology, vol. 103, no. 3, pp. 323-332, 2008.

[52] D. M. DiPasquale, R. J. Bloch, and R. M. Lovering, "Determinants of the repeated-bout effect after lengthening contractions," American Journal of Physical and Medicine Rehabilitation, vol. 90, no. 10, pp. 816-824, 2011.

[53] G. Y. Millet and R. Lepers, "Alterations of neuromuscular function after prolonged running, cycling and skiing exercises," Sports Medicine, vol. 34, no. 2, pp. 105-116, 2004.

[54] R. M. Enoka, "Eccentric contractions require unique activation strategies by the nervous system," Journal of Applied Physiology, vol. 81, no. 6, pp. 2339-2346, 1996.

[55] S. H. Westing, A. G. Cresswell, and A. Thorstensson, "Muscle activation during maximal voluntary eccentric and concentric knee extension," European Journal of Applied Physiology and Occupational Physiology, vol. 62, no. 2, pp. 104-108, 1991. 
[56] E. Kellis and V. Baltzopoulos, "Muscle activation differences between eccentric and concentric isokinetic exercise," Medicine and Science in Sports and Exercise, vol. 30, no. 11, pp. 1616-1623, 1998.

[57] R. M. Enoka, Neuromechanics of Human Movementedition, Human Kinetics Books, Champaign, Ill, USA, 3rd edition, 2002.

[58] D. A. Jones, D. J. Newham, and C. Torgan, "Mechanical influences on long-lasting human muscle fatigue and delayed-onset pain," The Journal of Physiology, vol. 412, pp. 415-427, 1989.

[59] U. Proske and D. L. Morgan, "Muscle damage from eccentric exercise: mechanism, mechanical signs, adaptation and clinical applications," The Journal of Physiology, vol. 537, no. 2, pp. 333345, 2001.

[60] D. G. Allen and H. Westerblad, "Role of phosphate and calcium stores in muscle fatigue," The Journal of Physiology, vol. 536, no. 3, pp. 657-665, 2001.

[61] R. Child, S. Brown, S. Day, A. Donnelly, H. Roper, and J. Saxton, "Changes in indices of antioxidant status, lipid peroxidation and inflammation in human skeletal muscle after eccentric muscle actions," Clinical Science, vol. 96, no. 1, pp. 105-115, 1999.

[62] K. Nosaka and P. M. Clarkson, "Muscle damage following repeated bouts of high force eccentric exercise," Medicine and Science in Sports and Exercise, vol. 27, no. 9, pp. 1263-1269, 1995.

[63] M. P. McHugh, "Recent advances in the understanding of the repeated bout effect: the protective effect against muscle damage from a single bout of eccentric exercise," Scandinavian Journal of Medicine and Science in Sports, vol. 13, no. 2, pp. 88-97, 2003.

[64] M. P. McHugh, D. A. J. Connolly, R. G. Eston, and G. W. Gleim, "Exercise-induced muscle damage and potential mechanisms for the repeated bout effect," Sports Medicine, vol. 27, no. 3, pp. 157-170, 1999.

[65] K. Nosaka and K. Sakamoto, "Effect of elbow joint angle on the magnitude of muscle damage to the elbow flexors," Medicine and Science in Sports and Exercise, vol. 33, no. 1, pp. 22-29, 2001.

[66] T. C. Chen, K. Nosaka, and P. Sacco, "Intensity of eccentric exercise, shift of optimum angle, and the magnitude of repeatedbout effect," Journal of Applied Physiology, vol. 102, no. 3, pp. 992-999, 2007.

[67] M. J. Newton, G. T. Morgan, P. Sacco, D. W. Chapman, and K. Nosaka, "Comparison of responses to strenuous eccentric exercise of the elbow flexors between resistance-trained and untrained men," Journal of Strength and Conditioning Research, vol. 22, no. 2, pp. 597-607, 2008.

[68] T. C. Chen, H. L. Chen, M. J. Lin, C. J. Wu, and K. Nosaka, "Potent protective effect conferred by four bouts of lowintensity eccentric exercise," Medicine and Science in Sports and Exercise, vol. 42, no. 5, pp. 1004-1012, 2010.

[69] G. Howatson, K. Van Someren, and T. Hortobágyi, "Repeated bout effect after maximal eccentric exercise," International Journal of Sports Medicine, vol. 28, no. 7, pp. 557-563, 2007.

[70] T. C. Chen and K. Nosaka, "Responses of elbow flexors to two strenuous eccentric exercise bouts separated by three days," Journal of Strength and Conditioning Research, vol. 20, no. 1, pp. 108-116, 2006.

[71] G. L. Warren, K. M. Hermann, C. P. Ingalls, M. R. Masselli, and R. B. Armstrong, "Decreased EMG median frequency during a second bout of eccentric contractions," Medicine and Science in Sports and Exercise, vol. 32, no. 4, pp. 820-829, 2000.

[72] G. Howatson and K. A. Van Someren, "Ice massage: effects on exercise-induced muscle damage," Journal of Sports Medicine and Physical Fitness, vol. 43, no. 4, pp. 500-505, 2003.
[73] K. Nosaka, M. Newton, P. Sacco, D. Chapman, and A. Lavender, "Partial protection against muscle damage by eccentric actions at short muscle lengths," Medicine and Science in Sports and Exercise, vol. 37, no. 5, pp. 746-753, 2005.

[74] D. J. Newham, D. A. Jones, and P. M. Clarkson, "Repeated highforce eccentric exercise: effects on muscle pain and damage," Journal of Applied Physiology, vol. 63, no. 4, pp. 1381-1386, 1987.

[75] H. S. Thompson, P. M. Clarkson, and S. P. Scordilis, "The repeated bout effect and heat shock proteins: intramuscular HSP27 and HSP70 expression following two bouts of eccentric exercise in humans," Acta Physiologica Scandinavica, vol. 174, no. 1, pp. 47-56, 2002.

[76] T. M. Lehti, R. Kalliokoski, and J. Komulainen, "Repeated bout effect on the cytoskeletal proteins titin, desmin, and dystrophin in rat skeletal muscle," Journal of Muscle Research and Cell Motility, vol. 28, no. 1, pp. 39-47, 2007.

[77] R. B. Child, J. M. Saxton, and A. E. Donnelly, "Comparison of eccentric knee extensor muscle actions at two muscle lengths on indices of damage and angle-specific force production in humans," Journal of Sports Sciences, vol. 16, no. 4, pp. 301-308, 1998.

[78] S. V. Brooks and J. A. Faulkner, "Severity of contraction-induced injury is affected by velocity only during stretches of large strain," Journal of Applied Physiology, vol. 91, no. 2, pp. 661-666, 2001.

[79] M. J. Falvo, B. K. Schilling, R. J. Bloomer, and W. A. Smith, "Repeated bout effect is absent in resistance trained men: an electromyographic analysis," Journal of Electromyography and Kinesiology, vol. 19, no. 6, pp. e529-e535, 2009.

[80] K. Nosaka, K. Sakamoto, M. Newton, and P. Sacco, "How long does the protective effect on eccentric exercise-induced muscle damage last?" Medicine and Science in Sports and Exercise, vol. 33, no. 9, pp. 1490-1495, 2001.

[81] P. M. Clarkson, K. Nosaka, and B. Braun, "Muscle function after exercise-induced muscle damage and rapid adaptation," Medicine and Science in Sports and Exercise, vol. 24, no. 5, pp. 512-520, 1992.

[82] R. G. Eston, S. Finney, S. Baker, and V. Baltzopoulos, "Muscle tenderness and peak torque changes after downhill running following a prior bout of isokinetic eccentric exercise," Journal of Sports Sciences, vol. 14, no. 4, pp. 291-299, 1996.

[83] D. L. Macintyre, W. D. Reid, D. M. Lyster, I. J. Szasz, and D. C. Mckenzie, "Presence of WBC, decreased strength, and delayed soreness in muscle after eccentric exercise," Journal of Applied Physiology, vol. 80, no. 3, pp. 1006-1013, 1996.

[84] R. Molina and B. S. Denadai, "Dissociated time course recovery between rate of force development and peak torque after eccentric exercise," Clinical Physiology and Functional Imaging, vol. 32, no. 3, pp. 179-184, 2012.

[85] L. C. R. Lima and B. S. Denadai, "The repeated bout effect: a comparison between upper and lower limbs," Motriz, vol. 17, no. 4, pp. 738-747, 2011.

[86] R. Molina and B. S. Denadai, "Muscle damage slows oxygen uptake kinetics during moderate-intensity exercise performed ate high pedal rate," Applied Physiology Nutrition and Metabolism, vol. 36, no. 6, pp. 848-855, 2011.

[87] P. Vassilis, B. Vassilios, M. Vassilis et al., "Isokinetic eccentric exercise of quadriceps femoris does not affect running economy," Journal of Strength and Conditioning Research, vol. 22, no. 4, pp. 1222-1227, 2008.

[88] K. E. Scott, R. Rozenek, A. C. Russo, J. A. Crussemeyer, and M. G. Lacourse, "Effects of delayed onset muscle soreness 
on selected physiological responses to submaximal running," Journal of Strength and Conditioning Research, vol. 17, no. 4, pp. 652-658, 2003.

[89] H. Kyrolainen, T. Pullinen, R. Candau, J. Avela, P. Huttunen, and P. V. Komi, "Effects of marathon running on running economy and kinematics," European Journal of Applied Physiology, vol. 82, no. 4, pp. 297-304, 2000.

[90] G. A. Gaesser and D. C. Poole, "The slow component of oxygen uptake kinetics in humans," Exercise and Sport Sciences Reviews, vol. 24, pp. 35-71, 1996.

[91] D. T. Cannon, A. C. White, M. F. Andriano, F. W. Kolkhorst, and H. B. Rossiter, "Skeletal muscle fatigue precedes the slow component of oxygen uptake kinetics during exercise in humans," The Journal of Physiology, vol. 589, no. 3, pp. 727-739, 2011.

[92] J. Hamill, P. S. Freedson, P. M. Clarkson, and B. Braun, "Muscle soreness during running: biomechanical and physiological considerations," Journal of Applied Biomechanics, vol. 7, no. 2, pp. 125-137, 1991.

[93] W. A. Braun and D. J. Dutto, "The effects of a single bout of downhill running and ensuing delayed onset of muscle soreness on running economy performed $48 \mathrm{~h}$ later," European Journal of Applied Physiology, vol. 90, no. 1-2, pp. 29-34, 2003.

[94] T. C. Chen, K. Nosaka, and J. H. Tu, "Changes in running economy following downhill running," Journal of Sports Sciences, vol. 25, no. 1, pp. 55-63, 2007.

[95] R. G. Eston, J. Mickleborough, and V. Baltzopoulos, "Eccentric activation and muscle damage: biomechanical and physiological considerations during downhill running," British Journal of Sports Medicine, vol. 29, no. 2, pp. 89-94, 1995.

[96] H. J. Appell, J. M. C. Soares, and J. A. R. Duarte, "Exercise, muscle damage and fatigue," Sports Medicine, vol. 13, no. 2, pp. $108-115,1992$.

[97] G. Y. Millet, K. Tomazin, S. Verges et al., "Neuromuscular consequences of an extreme mountain ultra-marathon," PLoS ONE, vol. 6, no. 2, Article ID e17059, 2011.

[98] T. C. Chen, K. Nosaka, and C. C. Wu, "Effects of a 30-min running performed daily after downhill running on recovery of muscle function and running economy," Journal of Science and Medicine in Sport, vol. 11, no. 3, pp. 271-279, 2008.

[99] S. A. Hahn, L. F. Ferreira, J. B. Williams et al., "Downhill treadmill running trains the rat spinotrapezius muscle," Journal of Applied Physiology, vol. 102, no. 1, pp. 412-416, 2007.

[100] B. C. Pereira, L. A. Filho, G. F. Alves et al., "A new overtraining protocol for mice based on downhill running sessions," Clinical and Experimental Pharmacology and Physiology, vol. 39, no. 9, pp. 793-798, 2012.

[101] R. Lynn and D. L. Morgan, "Decline running produces more sarcomeres in rat vastus intermedius muscle fibers than does incline running," Journal of Applied Physiology, vol. 77, no. 3, pp. 1439-1444, 1994.

[102] B. Essen, "Glycogen depletion of different fibre types in human skeletal muscle during intermittent and continuous exercise," Acta Physiologica Scandinavica, vol. 103, no. 4, pp. 446-455, 1978.

[103] D. A. Jones, D. J. Newham, J. M. Round, and S. E. J. Tolfree, "Experimental human muscle damage: morphological changes in relation to other indices of damage," The Journal of Physiology, vol. 375, pp. 435-448, 1986.

[104] T. C. Chen, "Effects of a second bout of maximal eccentric exercise on muscle damage and electromyographic activity," European Journal of Applied Physiology, vol. 89, no. 2, pp. 115121, 2003.
[105] W. C. Byrnes, P. M. Clarkson, J. S. White et al., "Delayed onset muscle soreness following repeated bouts of downhill running," Journal of Applied Physiology, vol. 59, no. 3, pp. 710-715, 1985.

[106] T. C. Chen, H. L. Chen, C. J. Wu et al., "Changes in running economy following a repeated bout of downhill running," Journal of Exercise Science and Fitness, vol. 5, no. 2, pp. 109-117, 2007.

[107] G. Howatson, M. P. McHugh, J. A. Hill et al., "Influence of tart cherry juice on indices of recovery following marathon running," Scandinavian Journal of Medicine and Science in Sports, vol. 20, no. 6, pp. 843-852, 2010.

[108] K. S. Kuehl, E. T. Perrier, D. L. Elliot, and J. C. Chesnutt, "Efficacy of tart cherry juice in reducing muscle pain during running: a randomized controlled trial," Journal of the International Society of Sports Nutrition, vol. 7, article 17, 2010. 

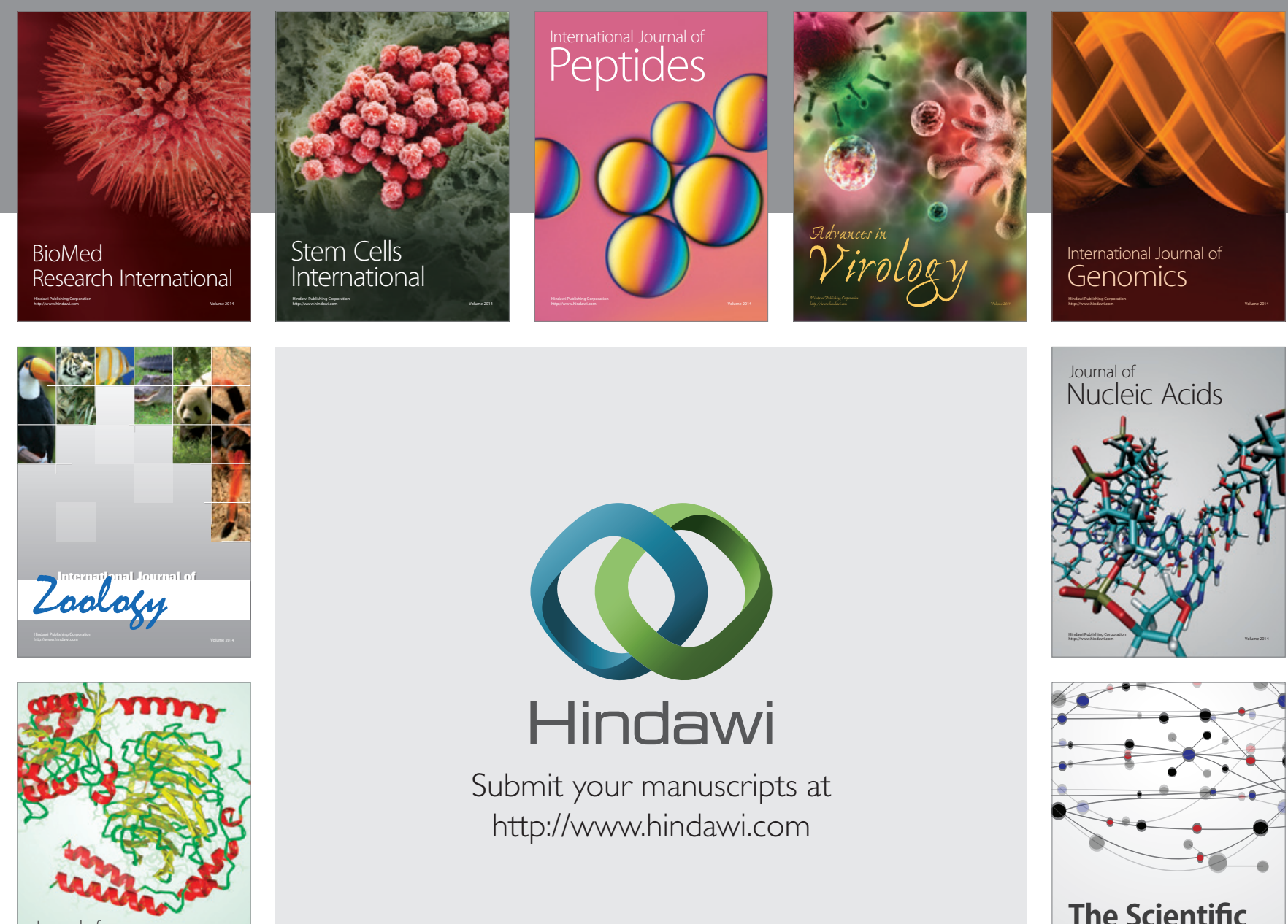

Submit your manuscripts at

http://www.hindawi.com

Journal of
Signal Transduction
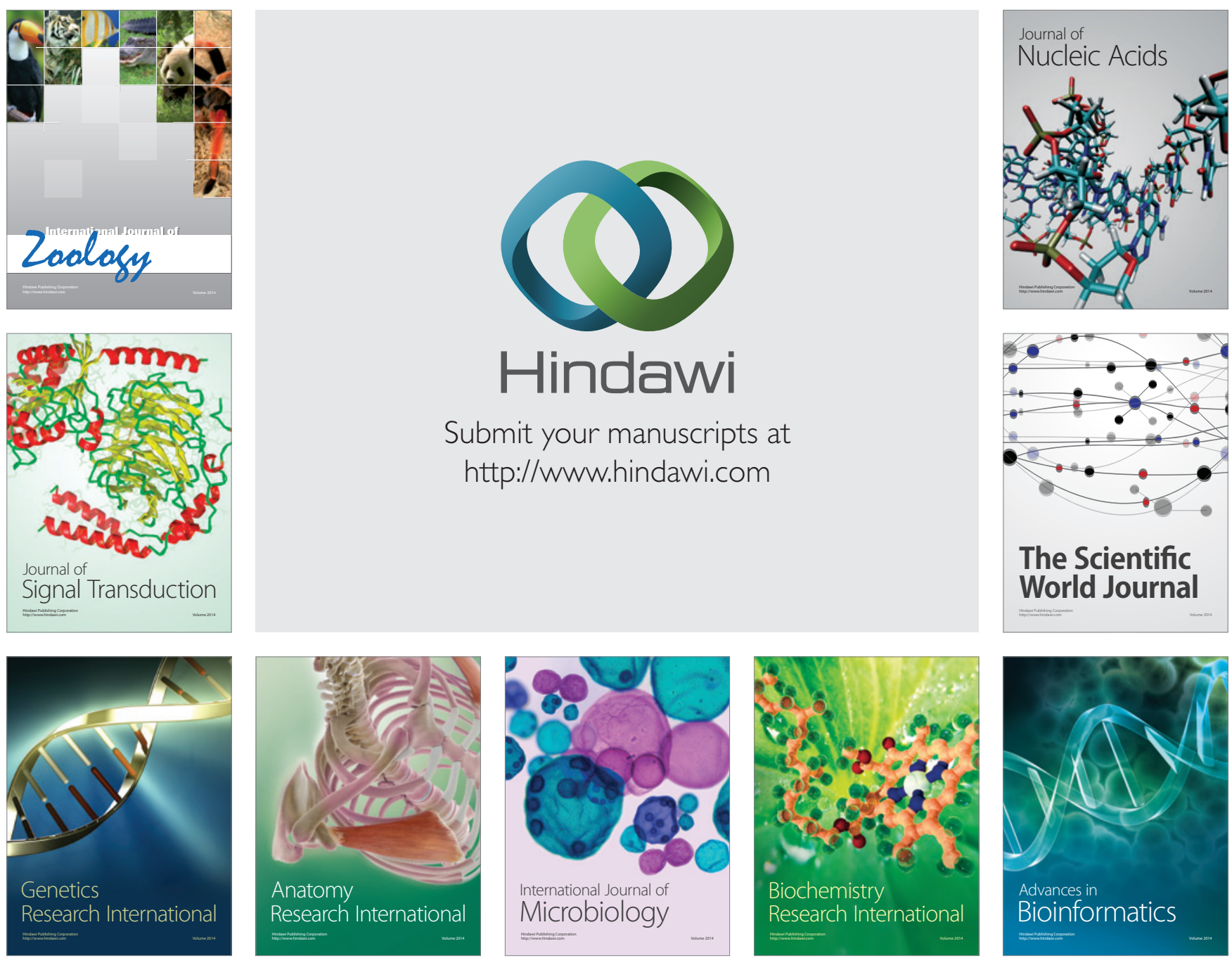

The Scientific World Journal
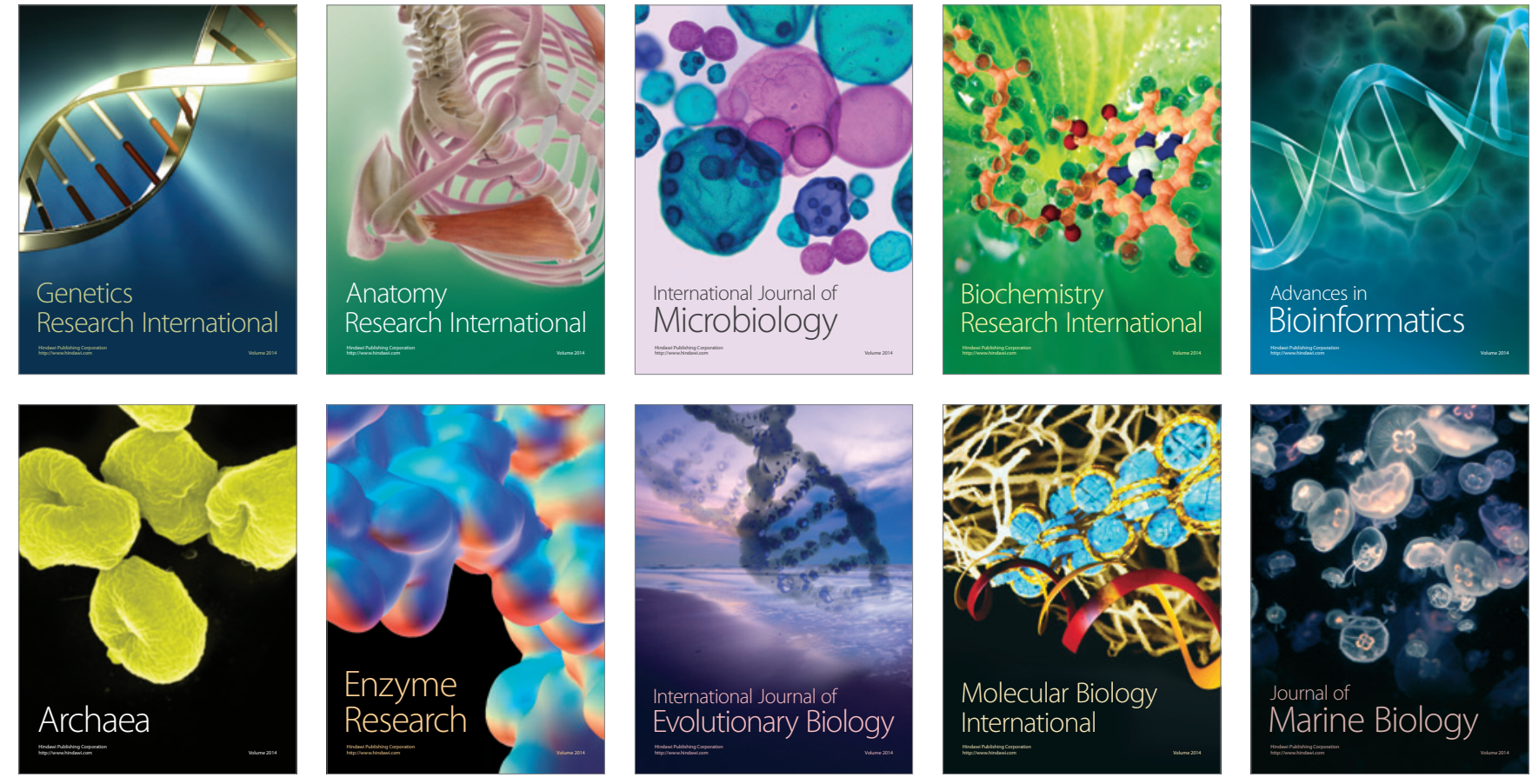